Method The workshop was delivered through a blend of plenary and small group sessions modelled on the CEBM approach to teaching EBP. In all plenary's speakers used real life examples from their own experience with consistent emphasis on the impact to patients and the importance of patient values.

The programme for day one covered the Ask and Search domains. Day two explored appraisal of different study designs. Day three focused on putting evidence into practice with plenaries on

- From Evidence to recommendations

- Shared decision making

- Implementation planning

These topics were chosen to reflect the real-word challenges health care professionals face. Each small group was required to demonstrate the skills attained including, asking an answerable question in PICO format, finding the evidence, critical appraisal of the best evidence and how they would put the evidence into practice in the real-world setting

Results The small group presentations covered a wide range of topics which were based on a real clinical query of a participant. Each group discussed the quality of the evidence to answer their question, the potential benefit and harm, patient preferences and values and resources required before presenting their recommendations. They also described barriers and facilitators to the implementation of their recommendations.

In the evaluation form all participants rated their ability to practice EBP a 4-5 on a five-point scale compared with a range of $2-5$ in a previous workshop. When asked what aspects of the course were most useful, comments included;

'Practical aspects of bringing EBP to patients'

'Guidelines to aid implementation'

'Connection of theory to real world practice'

'Shared decision making'

'Apply information in practice'

Conclusions Our goal in EBPI is to promote the practice of EBP throughout the healthcare system in Ireland to improve patient outcomes. To be successful we need health care professionals to understand the principles of EBP. Much work internationally has gone into addressing the skills needed to ask focused questions, search the literature and appraise the literature for its quality and applicability. However, there is less agreement on how to teach integrating the best evidence with clinical expertise and patient values and applying it in practice. By developing this 'evidence into practice' module we are addressing the curriculum gap in how to teach the 'apply in practice' domain of EBP while building capacity and leadership in EBP in Ireland.

\section{EFFECT OF AN EBP LEARNING PROGRAM ON CONFIDENCE AND COMPETENCE IN EBP}

Michael Pianta, Edward Nguyen, Laura Downie. The University of Melbourne, Melbourne, Australia

\subsection{6/bmjebm-2019-EBMLive.47}

Objectives The aim of this study was to investigate the effect of an EBP learning program on learner confidence and competence in the use of EBP.
Method The Evidence-Based Practice Confidence (EPIC) scale (Salbach and Jaglal, J Eval Clin Pract, 2011) and the Assessing Competency in EBM (ACE) tool (Ilic et al, BMC Med Ed, 2014) were used to measure learner $(n=49)$ competence and confidence before and after a one-semester EBP learning program that incorporated the CrowdCARE platform.

Results The program resulted in a significant increase in both confidence $(+23.0 \%, 95 \%$ CI $[+18.2 \%,+27.8 \%])$ and competence $(+38.0 \%, 95 \%$ CI $[+33.3 \%,+42.8 \%])$ in the use of EBP. The largest increases in confidence were in critically appraising measurement properties, interpreting statistical tests, and integrating research evidence, clinical judgment and patient preferences; the largest increases in competence were in asking an answerable question and appraising the evidence. The smallest increases in confidence were in identifying gaps in knowledge and conducting a literature search; the smallest increase in competence was in applying the evidence.

Conclusions The learning program was effective at improving confidence and competence in EBP. The use of the EPIC scale and ACE tool allows identification of areas for improvement.

\section{PHARMACOLOGIC TREATMENT OF MAJOR DEPRESSION: MAKING RESEARCH EVIDENCE AVAILABLE WITH A PEER-REVIEWED ALGORITHM IN AN EASY TO USE COMPUTER PROGRAM}

${ }^{1,2}$ David Osser, ${ }^{1,3}$ Robert Patterson. ${ }^{1}$ Harvard Medical School, Boston, MA, USA; ${ }^{2}$ VA Boston HealthCare System, Brockton Division, Brockton, MA, USA; ${ }^{3}$ McLean Hospital, Belmont, MA, USA

\subsection{6/bmjebm-2019-EBMLive.48}

Objectives The Psychopharmacology Algorithm Project at the Harvard South Shore Psychiatry Department has been preparing evidence-derived algorithms for choosing medication for psychiatric disorders for 25 years. They provide clinicians with syntheses of the best evidence for efficacy, effectiveness, safety, and cost of pharmaceuticals in a format that is more prescriptive than guidelines. The aim is to present very specific and very context sensitive best practice. The algorithms start with recommendations for medication-naive patients. After that, suggestions for patients who had one failed trial are offered, and then we address deeper levels of treatmentresistance. Important comorbidity is considered. They are usable on cell phones. Practitioners can obtain an actionable recommendation in 1-2 minutes. We will demonstrate the major depression algorithm, most recently published in the February 2019 issue of Harvard Review of Psychiatry.

Method Small groups of clinicians create new algorithms or update older ones and then submit papers describing them for peer review in psychiatric journals. The review process involves reaching consensus with the expert reviewers who may not agree with all the interpretations of the evidence leading to the recommendations in the initial draft. This review process enhances the validity of the final version. After acceptance, the authors construct the on-line versions of the algorithms. Users see the entire flowchart and click on the node representing their patient's current clinical status - ranging from first use of medication to very treatment resistant, while considering the impact of various illness subtypes and comorbidities. The website (www.psychopharm.mobi) currently has 9 algorithms. All algorithms emphasize that they advise on choice of pharmacotherapy only, but non-medication approaches are valid, important, and may be preferred. 